\title{
Use of carbon nanotube sensor for detecting postoperative abnormal respiratory waveforms
}

\author{
Masashi Kobayashi ${ }^{1}$, Yohei Wada ${ }^{2}$, Yasuro Okumiya ${ }^{2}$, Koji Yataka ${ }^{2}$, Katsunori Suzuki ${ }^{2}$, \\ Yasuhiro Nakashima ${ }^{1}$, Hironori Ishibashi ${ }^{1}$, Kenichi Okubo ${ }^{1}$ \\ ${ }^{1}$ Department of Thoracic Surgery, Tokyo Medical and Dental University, Tokyo, Japan; ${ }^{2}$ Research and Development Division, Technology Unit, \\ Yamaha Corporation, Hamamatsu, Japan \\ Contributions: (I) Conception and design: M Kobayashi, K Okubo; (II) Administrative support: Y Wada, Y Okumiya, K Yataka, K Suzuki; (III) \\ Provision of study materials or patients: M Kobayashi, K Okubo; (IV) Collection and assembly of data: M Kobayashi, H Ishibashi, Y Nakashima, K \\ Okubo; (V) Data analysis and interpretation: M Kobayashi; (VI) Manuscript writing: All authors; (VII) Final approval of manuscript: All authors. \\ Correspondence to: Masashi Kobayashi. 1-5-45 Yushima, Bunkyo-ku, Tokyo 113-8519, Japan. Email: mkobthsr@tmd.ac.jp.
}

Background: This feasibility study aimed to detect respiratory waveforms from thoracic movements and evaluate if postoperative complications could be predicted using a carbon nanotube sensor.

Methods: Fifty patients who underwent lung resection for lung tumors were enrolled. The lung monitoring system of the carbon nanotube sensor was placed on bilateral chest walls across the $6^{\text {th }}-9^{\text {th }}$ ribs to measure chest wall motion. We examined the respiratory waveform in relation to surgical findings, postoperative course, and complications using Hilbert transform and Fast Fourier Transform (FFT).

Results: Of 50 patients (37 males, 13 females), 22 were included in the normal lung function group and 28 were included in the low lung function group. The respiratory rate and waveform indicated a regular pattern in the normal lung function group and the respiratory rate could be detected. Conversely, irregular respiratory pattern was detected in $70 \%$ of patients in the low lung function group. There was no significant different overall envelope peak value between operated side and non-operated side $(0.195 \pm 0.05$ and $0.18 \pm 0.06)$. In contrast, there was significantly high peak value in the presence of postoperative complications $(\mathrm{P}<0.05)$. And there was a significantly higher peak value in air leakage presence than air leakage absence in operated side $(\mathrm{P}=0.045)$.

Conclusions: The present study confirmed the feasibility of the sensor. It is promising in visualizing the respiratory state and detecting respiratory changes postoperatively.

Keywords: Respiratory waveform; stretchable sensor; Fourier transform

Submitted Jan 24, 2021. Accepted for publication Apr 14, 2021.

doi: $10.21037 /$ jtd-21-156

View this article at: http://dx.doi.org/10.21037/jtd-21-156

\section{Introduction}

In recent years, the number of patients with low lung function has increased significantly in the field of thoracic surgery (1-3). Lung resection in patients with low lung function requires a physical reduction of the lung parenchyma. The range of lung resection is dependent on the balance between the lung volume to be resected and the residual lung function (4).

Patients with low pulmonary function due to emphysema or interstitial pneumonia have a high frequency of postoperative complications, which may be severe (5-7). Prevention and early detection of postoperative respiratory complications are urgent issues. Until now, the less

\footnotetext{
^ ORCID: 0000-0002-1450-0589.
} 

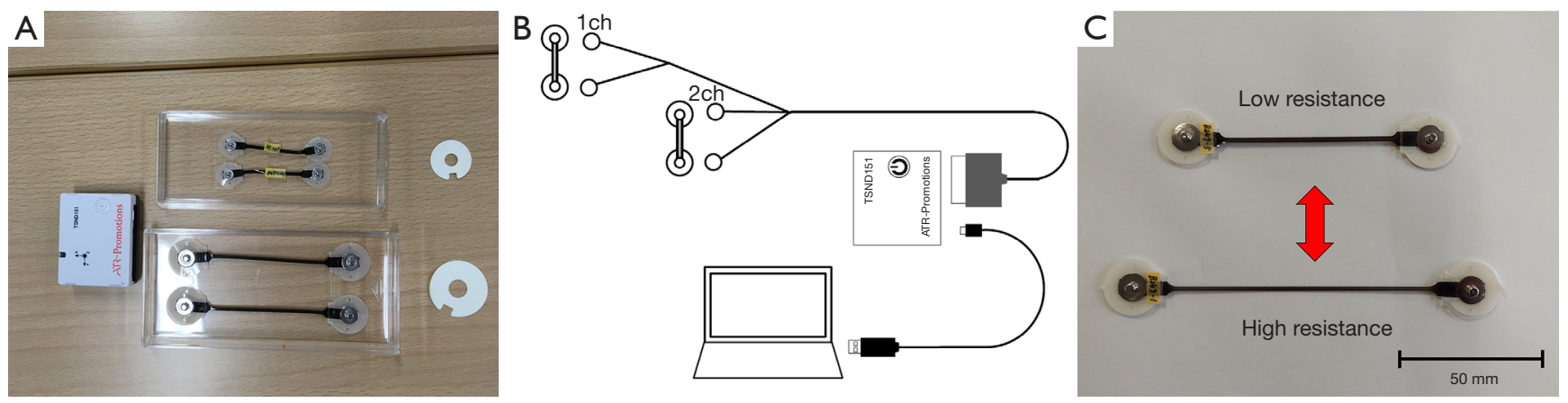

Figure 1 Stretchable carbon nanotube sensor system. (A) Schema of stretchable sensor system, 1ch: right chest wall, 2ch: left chest wall; (B) nano-structure change of CNT; (C) working mechanism of the CNT sensor. CNT, carbon nanotube.

invasive pulse oximeter has been used as the standard for postoperative respiratory monitoring. However, in recent years, using oxygenation alone as an evaluation index has become increasingly insufficient in detecting respiratory failure postoperatively. In other words, a device that monitors ventilation is preferable to assess the respiratory state accurately in the acute phase. Ventilation monitoring technology can be used to evaluate the respiratory state based on the measurement of thoracic impedance (8), respiratory sound patterns (9), and imaging (10). Recently, the review by Tukanova et al. reported the usefulness of that the respiratory inductive plethysmography and compartmental analysis of the chest wall volume by means of an optoelectronic plethysmography (11). However, there is no effective device or objective index for such postoperative monitoring.

The stretchable sensor developed by Yamaha Corporation (GummiStra ${ }^{\mathrm{TM}}$, Yamaha Corporation, Hamamatsu, Japan) consists of a special fiber that acts as a strain sensor (12). It is attached to an arbitrary body surface in a tight seal. Target muscle movement is measured based on the stretched width of the fiber and is continuously digitally processed and recorded. By placing sensors on both sides of the rib cage, it is possible to directly measure and record the ventilation state, breathing rate, and breathing depth. This makes it easy to compare postoperative respiratory states, which was not possible before. The stretchable sensor itself is small and easy to handle and is similar to an electrocardiogram or pulse oximeter used in conventional postoperative management. We performed this feasibility study to assess thoracic maximum movement width using the stretchable sensor.

We present the following article in accordance with the MDAR checklist (available at http://dx.doi.org/10.21037/ jtd-21-156).

\section{Methods}

\section{Materials}

A stretchable sensor was provided by Yamaha (Yamaha Corporation). This sensor detects elongation and contraction of material. Change in electric resistance is measured using nano structural changes in carbon nanotube (CNT) and with a multi-layered tube structure called multi wall CNT (MWCNT). The sensor is thin and flexible; its electrical resistance changes according to repeated stretching and shrinkage (Figure 1A). It can detect and record discrimination between small and large distortions. CNT-based stretchable sensors have excellent response and linear changes based on elongation-resistance relationship. The sensor length is $6 \mathrm{~cm}$.

\section{Monitoring system}

The schematic diagram of the monitoring system is shown in Figure $1 B, C$.

The postoperative monitoring system consists of the following two components: (1) the stretchable sensor and (2) personal computer with software for analysis of data.

\section{Recording and analysis}

We used python programming language to apply Hilbert transform and Fast Fourier Transform (FFT) to the respiratory waveform. Hilbert transform was analyzed using the scipy library (https://docs.scipy.org/doc/scipy/reference/ signal.html\#module-scipy.signal) and the Fourier transform 
was analyzed using the NumPy library (https://numpy.org/ doc/stable/reference/generated/numpy.fft.fft.html).

Hilbert transform: It is an operation that shifts the phase of all frequency components by $90^{\circ}$ relative to a given function. In this study, the respiratory waveform was phased by $90^{\circ}$ to create an envelope and a sine wave with and without amplitude fluctuation was derived from it. The respiratory waveform of the sine wave was adjusted so that the evaluation value approached 0 if there was no amplitude fluctuation and approached 1 if there was amplitude fluctuation.

FFT: It is an algorithm that converts a data signal in the time domain into a signal in the frequency domain. By

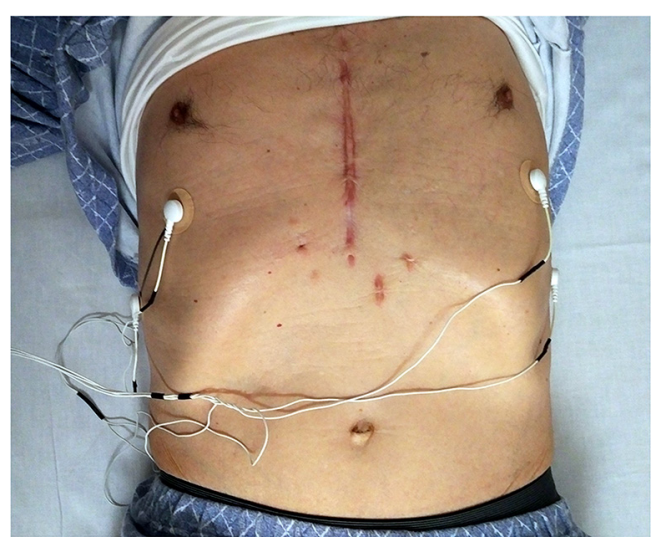

Figure 2 Photo image of stretchable sensor attachment site on chest wall. using FFT, the important characteristics of the signal-the frequency component-is clarified.

The lung monitoring system was placed on bilateral chest walls across the $6^{\text {th }}-9^{\text {th }}$ ribs and chest wall motion was assessed preoperatively for more than $10 \mathrm{~min}$ and immediately after surgery. The monitoring was continued until the next morning (Figure 2).

Based on the accumulated data, the patients included in the study were divided into a normal lung function group and a low lung function group; a respiratory waveform and peak value of the frequency characteristics of the envelope in the respiratory pattern were extracted using FFT along with respiratory pattern using Hilbert transformation.

The data were processed using a finite impulse response (FIR) filter. Noise including the body position fluctuations and transient muscle tone was removed. The variables were adjusted so that only respiratory data remained (Figure 3). The adjusted data were confirmed with the respiratory pattern in each case.

Next, raw data were analyzed using the Hilbert transform. After removing the low-frequency range with a high-pass filter, the respiratory pattern was quantified. Subsequently, the respiratory pattern of the envelope was extracted.

Lastly, the maximum amplitude range of the frequency was calculated from the enveloped derived using FFT. We defined the peak value as the maximum value of the envelope. And the peak value picks up the maximum respiratory amplitude, as shown in the Figure $4 A$. In a normal respiratory state, the envelope is gradual curve
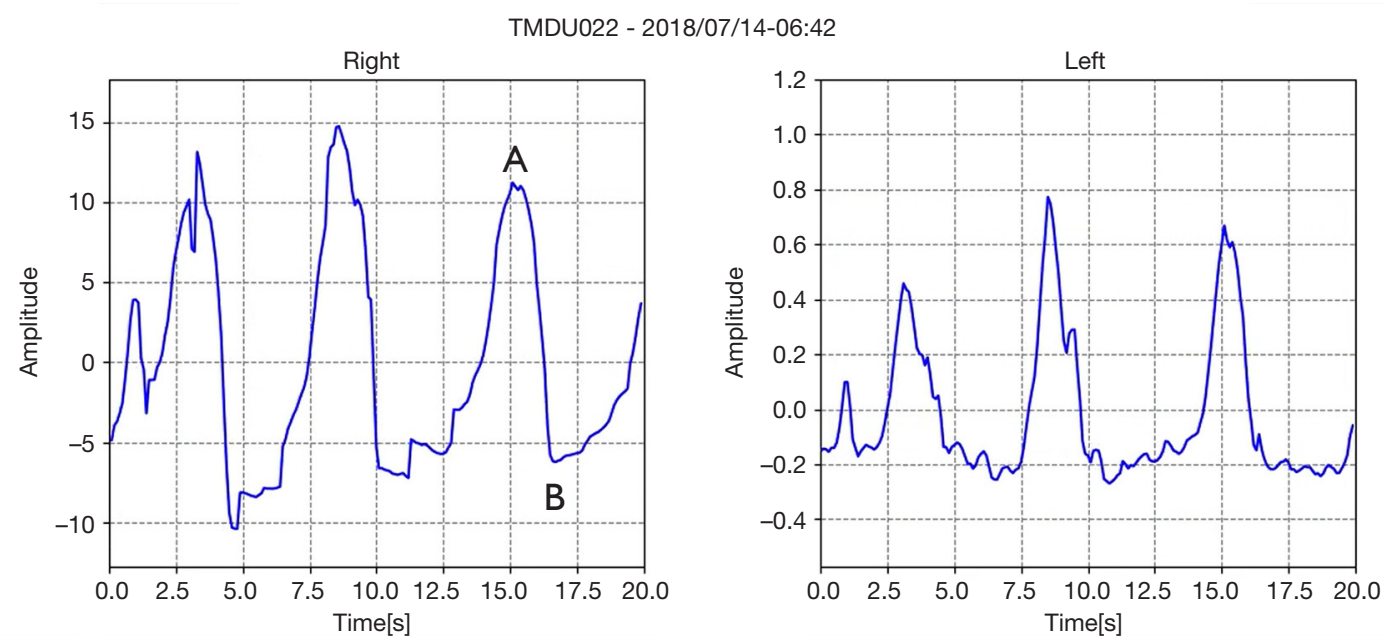

Figure 3 The relative change of resistance in chest motion from stretch to shrink. (A) Inhalation; (B) exhalation. 
A 18-09-07 01:00:00 - 2018-09-07 01:19:59/900000-Rch
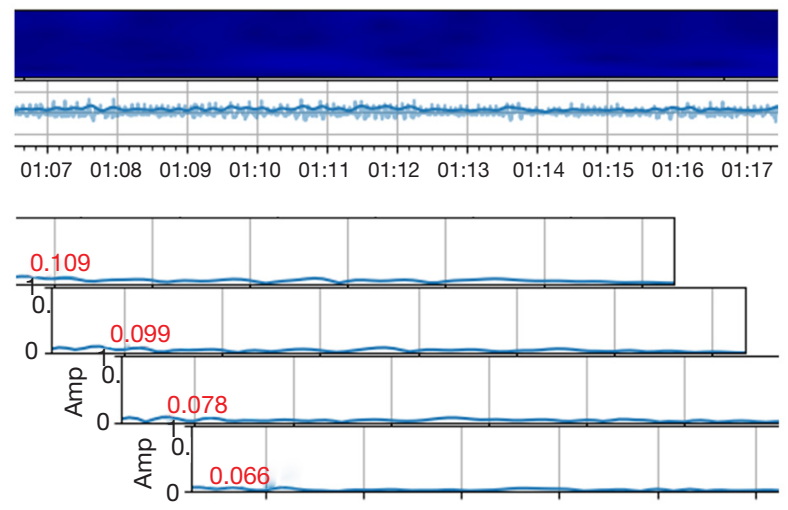

B TMDU030 - 2018-09-06 22:40:00 - 2018-09-06
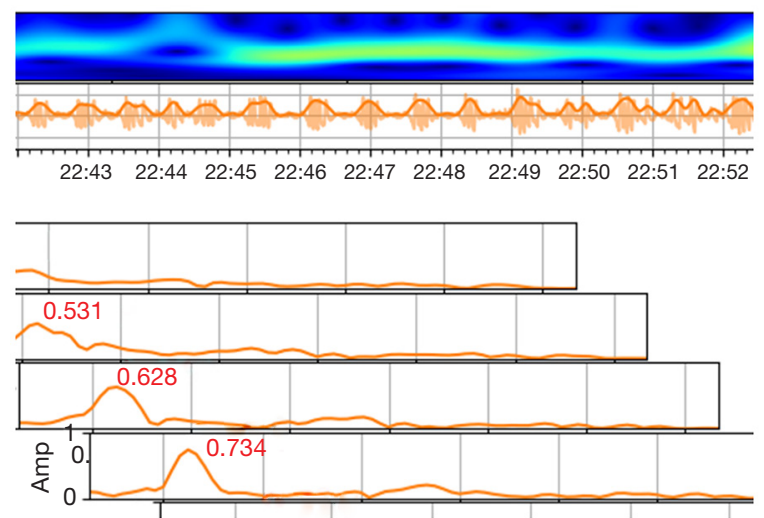

Figure 4 Schema of peak value as the maximum value of the envelope using FFT. Red arrow; peak value point. (A) The envelope of normal lung function was almost flat line. (B) The envelope of abnormal lung function had remarkable time fluctuation. FFT, fast fourier transform.

(Figure 4A). On the other hands, the abnormal respiratory state showed that the amplitude of the envelope curve is larger in the envelope due to the disturbance of the respiratory pattern (Figure 4B). Regarding the peak value, if the envelope is constant, the change in respiratory amplitude is small and if there is an envelope fluctuation, the change in respiratory amplitude is large. In other words, the amplitude change is large when there is a disorder of the respiratory system. We examined the relation between peak value and surgical factors by appropriate evaluation values.

\section{System evaluation}

The primary outcome in this feasibility study was the recording of inhalation and exhalation in bilateral lungs using the stretchable sensor. The secondary outcomes were to evaluate the relationship between (1) the surgical approach and chest wall motion, (2) chest wall motion in chronic respiratory disease and normal lung, (3) postoperative complications and the data of the stretchable sensor.

\section{Patients}

This study was approved by the ethics committee of the Tokyo Medical and Dental University in March 2018 (M2017-255). And the study was conducted in accordance with the Declaration of Helsinki (as revised 2013). Informed consent was obtained from all patients. Overall, 50 patients who underwent lung resection with segmentectomy or lobectomy for resectable lung tumors at Tokyo Medical and Dental University Hospital between September 2018 and April 2019 were enrolled. For surgical approach, open thoracotomy was selected for patients with low lung function and preoperative treatment. For segmentectomy, we included patients with pure GGO of $15 \mathrm{~mm}$ or less, or metastatic lung tumors or lung tumors that anatomically required segmentectomy.

We defined two groups using the following criteria; the criteria for normal lung function group were $\% \mathrm{VC} \geq 80$, $\% \mathrm{FEV} \geq 70$ with and without chronic obstructive pulmonary disease (COPD) or interstitial pneumonia (IP) patterns; and the criteria for the low lung function group were $\% \mathrm{VC}<80$ or $\% \mathrm{FEV}<70$ with/without COPD or IP patterns. This threshold was chosen because a $10 \%$ threshold corresponds to Global Initiative for Chronic Obstructive Lung Disease (GOLD) stage II or worse in patients with isolated COPD. Emphysema was defined as hyperlucent area of lung that lacked a distinct wall. Interstitial pneumonia (IP) was classified to $7^{\text {th }}$ criteria with pathological pattern according to the American Thoracic Society (ATS) and European Respiratory Society (ERS).

Pain management included non-steroidal antiinflammatory drugs, acetaminophen infusion, epidural anesthesia, or subcutaneous morphine patient-controlled analgesia in the postoperative period. The pain was controlled to a score of $0-2$ on the Numerical Rating Scale.

Regarding postoperative oxygen administration, $5 \mathrm{~L}$ $35 \%$ inspiron mask was used in all cases. Oxygen saturation $\left(\mathrm{SpO}_{2}\right)$ was maintained at $96-99 \%$ until the next morning. 
Table 1 List of postoperative complication related to chest wall motion

\begin{tabular}{ll}
\hline Complication & AE: $\geq$ grade Illa \\
\hline Air leakage & $>5$ days or Need treatment \\
Atelectasis & Need bronchoscopy \\
Pneumonia & Need bronchoscopy \\
Atrial fibrillation & Need treatment under local anesthesia \\
Bradyarrhythmia & Need treatment under local anesthesia \\
Schemic heart & Need a percutaneous coronary intervention \\
disease & \\
Subcutaneous & Need replacement of drain \\
emphysema & \\
Wound infection & Need treatment under local anesthesia \\
\hline
\end{tabular}

$\mathrm{AE}$, adverse event.

Neither case demonstrated a significant decrease in $\mathrm{SpO}_{2}$ under oxygen administration.

\section{Defined the postoperative complications}

Possible postoperative complication related to chest wall motion were defined as follow: air leakage, atelectasis, pneumonia, wound infection, pulmonary embolism, subcutaneous emphysema, ischemic heart disease, and bradyarrhythmia by the Clavien-Dindo classification (CTCAE5.0) and the STSGTSD guidelines (Table 1). Postoperative complications were analyzed to grade IIIa or higher within 30 days after surgery.

\section{Statistical analysis}

Univariate analysis included the sex, age, smoking, histology, lung function, surgical approach, surgical procedure, surgical side, recording time, respiratory rate, and postoperative complications in the lung function group. The values obtained from their correlation with the items was evaluated surgical approach, surgical procedure, and postoperative complications for each surgical side and nonoperative side. Cases of re-surgery of lung resection or partial lung resection were excluded. Statistical significance was assessed using $t$-test, paired $t$-test and Chi-squared test. A $\mathrm{P}$ value of $<0.05$ was considered to indicate statistical significance. All statistical analyses were performed using Stat View v5.0 (SAS Institute Inc.).

\section{Results}

Of 50 patients ( 37 males, 13 females), 22 were in the normal lung function group including one patient with COPD and 28 were in the low lung function group; of the latter, 21 had COPD and 7 had IP. Additionally, 17 underwent thoracotomy, 33 underwent video-assisted thoracic surgery, 38 underwent lobectomy, and 12 underwent segmentectomy. Postoperative complications were included air leak $(n=2)$ in the normal lung function group and atelectasis $(n=3)$ and air leak $(n=4)$ in the low lung function group (Table 2).

Sensor twisting and tension problems due to body movement over short terms were observed but there was no affected to analysis of respiratory and peak value.

\section{Analysis for respiratory waveform}

The average measurement time was 762 minutes and there were no complications with the stretchable sensor. The mean respiratory rate was $13.9 / \mathrm{min}$. There was no significant difference between the normal group (13.5/min) and the low lung function group (14.5/min).

The respiratory rate and waveform indicated a regular pattern in the normal group and the respiratory rate could be detected (Figure $5 A$ and $5 B$ ). Conversely, irregular respiratory pattern was detected in $70 \%$ of patients in the low lung function group (Figure $5 C$ and $5 D$ ).

\section{Analysis for peak value}

In the analysis of this study, regarding the overall peak value, the peak value of operated side was $0.195 \pm 0.05$ and $0.18 \pm 0.06$ in non-operated side, there was no significant different overall envelope peak value.

There was no significant different peak value in surgical approach, procedure relation to operated side and nonoperated side.

In contrast, there was a significantly high peak value in the presence of postoperative complications than in their absence relation to R-peak peak value, L-peak peak value, Non-operated side peak value $(0.173 \pm 0.05$ versus $0.222 \pm 0.1$, $0.173 \pm 0.06$ versus $0.225 \pm 0.041,0.168 \pm 0.06$ versus $0.217 \pm 0.07 ; \mathrm{P}=0.034,0.048,0.031$ ) (Table 3).

Regrading to the air leakage, the peak value of air leakage presence was $0.229 \pm 0.09$ and $0.179 \pm 0.05$ in air leakage absence at operated side, there was a significantly higher peak value in air leakage presence than air leakage absence 
Table 2 Clinical characteristics of patients

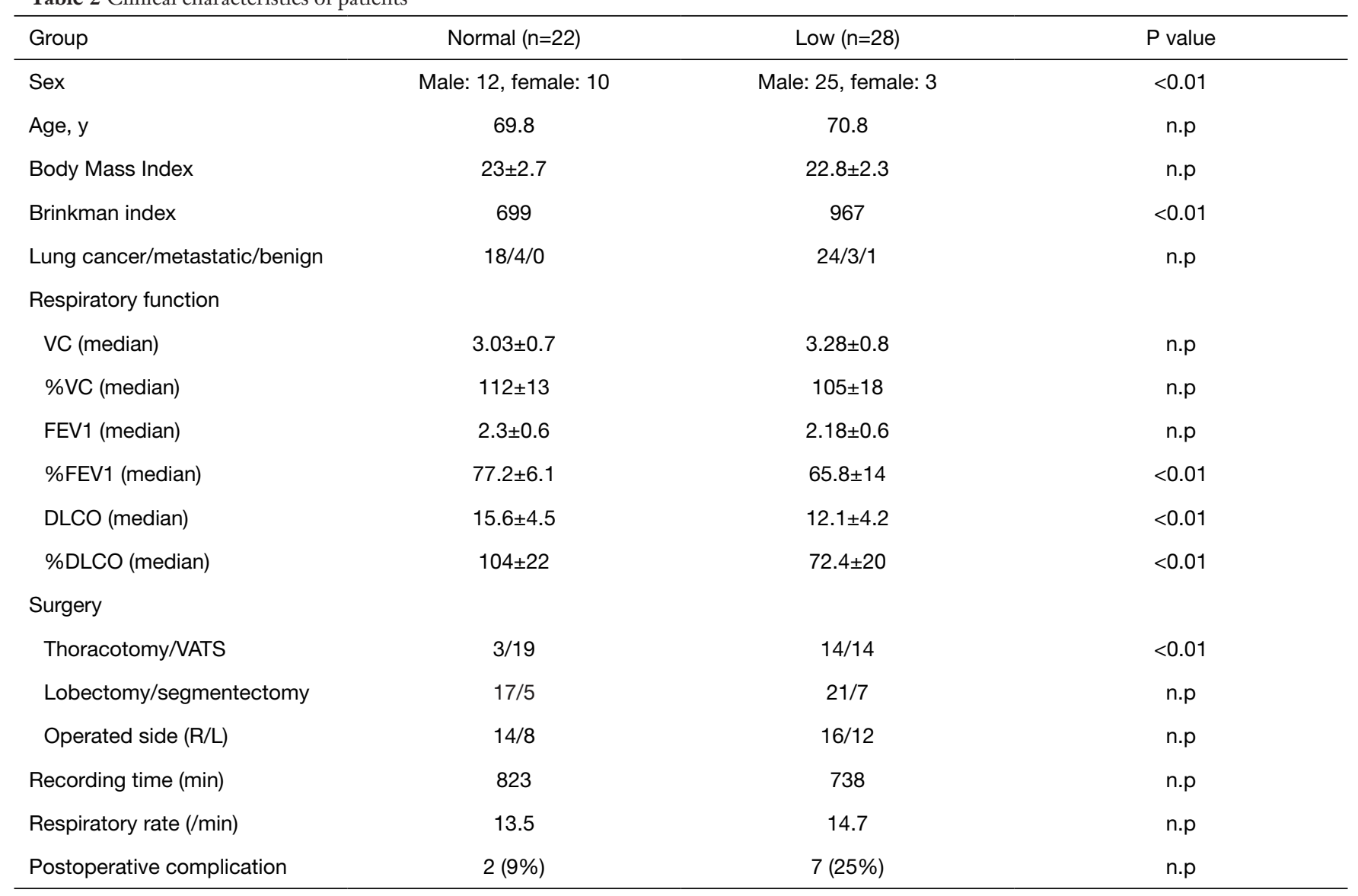

(Table 4).

Furthermore, there was no significant peak value with atelectasis relation to Operated side, non-operated side, R-peak peak value, L-peak peak value.

\section{Discussion}

Stretchable sensors have several applications and advantages (12-15). The sensor is made of CNT-based, stretchable, and durable material. It is small and lightweight and mounted with a seal; easy measurement is possible at any point. Based on the pilot study (data not shown), data were collected by measuring the respiratory waveform across the $6^{\text {th }}$ and $9^{\text {th }}$ ribs. The measurement began at the end of the surgery and lasted for more than 8 hours. The sensor could not measure for periods of time due to slacking or twisting of the sensor but data were collected as planned.

Several studies have evaluated respiratory sensors that measure laser position (16), entire thorax movement (17), and end-tidal $\mathrm{CO}_{2}$ (18). However, these are difficult methods to evaluate the respiratory pattern in clinical practice. Their sensitivity is poor in abdominal breathing, tachypnea, and superficial breathing. The problems also include facilities and man power as they lack versatility for clinical applications.

In the field of rehabilitation, the respiratory state is measured by the range of motion of the rib cage using a tape measure. This is a method for evaluating the difference in thoracic dilatation; it appears to represent lung and thoracic compliance and respiratory muscle movement. However, chest movements in a few cases are not consistent in the procedure and location using the tape measurement; however, the CNT sensor could assess the left and right chest walls separately and confirm the precise respiratory movements.

In the present study, we were able to measure respiratory waveforms in all patients and demonstrate characteristic waveforms in those in the low lung function group using 

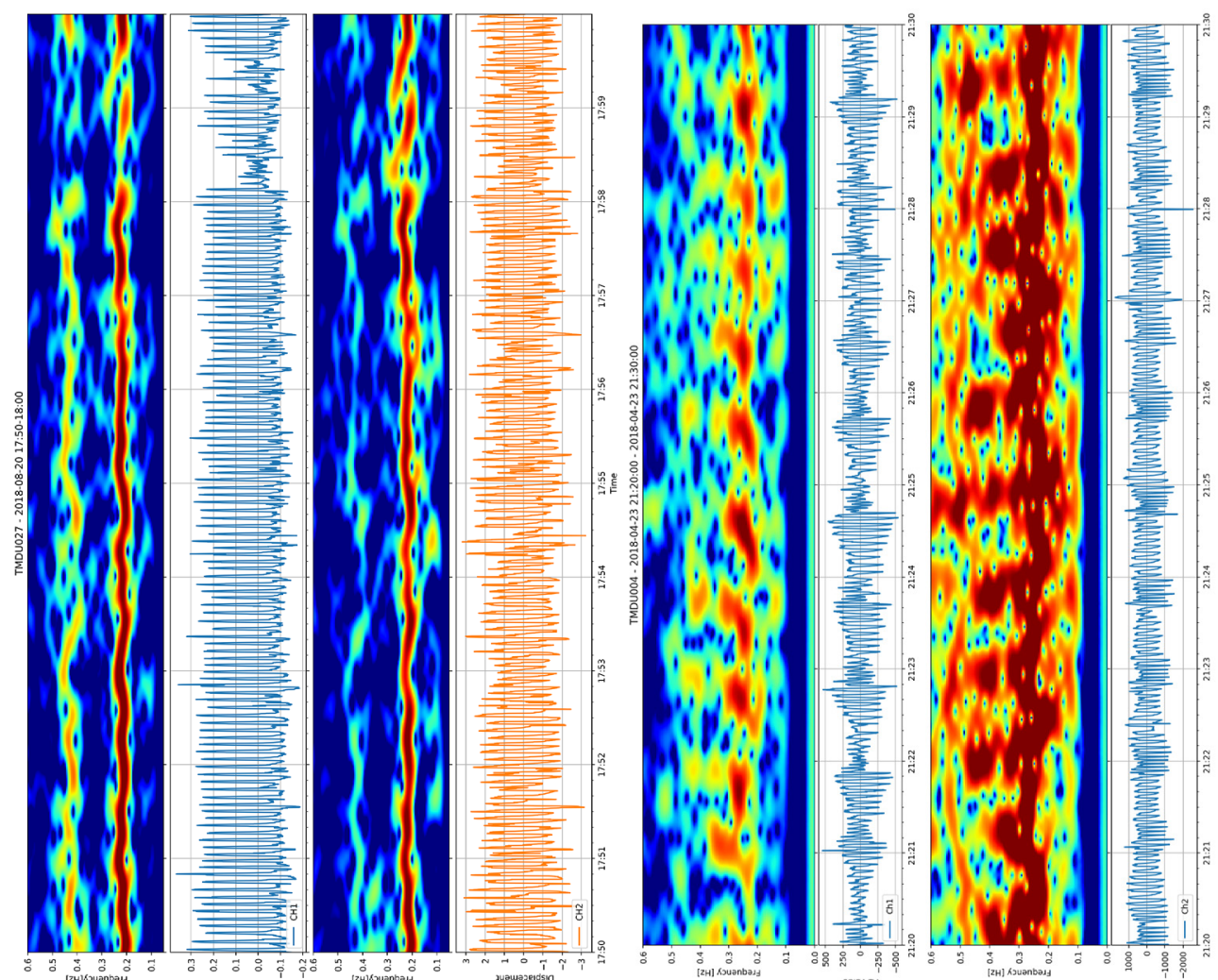

$\infty$
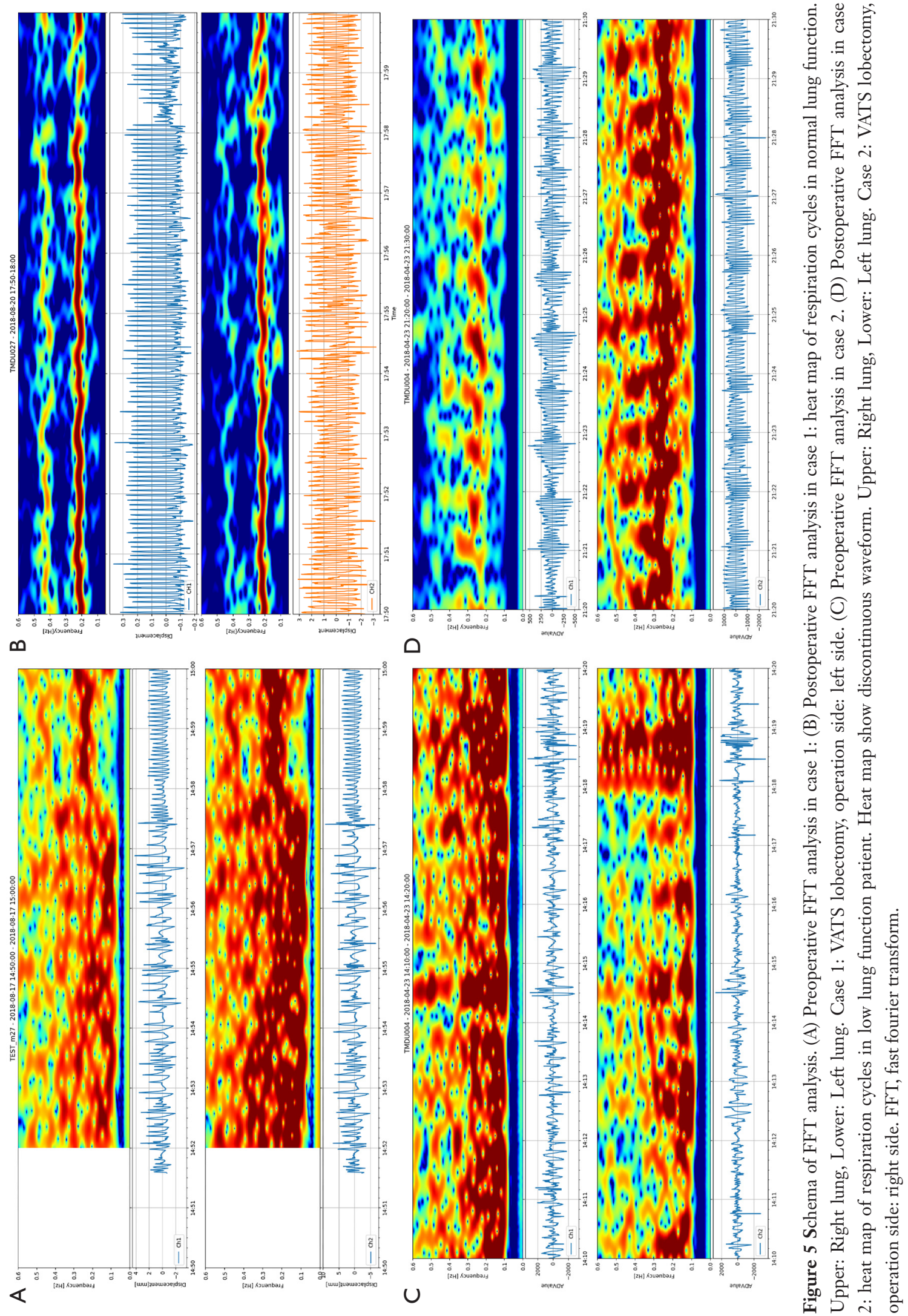
Table 3 Analysis of overall envelope peak value by Hilbert transform

\begin{tabular}{lccc} 
& Operated side & Non-operated side & P value \\
\hline Overall envelope peak value & $0.195 \pm 0.05$ & $0.18 \pm 0.06$ & n.p \\
Approach & Thoracotomy [17] & VATS [33] & 0.19 \\
Operated side & 0.186 & 0.174 & n.p \\
Non-operated side & 0.19 & Segmentectomy [12] & n.p \\
Procedure & Lobectomy [38] & 0.181 & n.p \\
Operated side & 0.19 & 0.178 & \\
Non-operated side & 0.184 & & \\
\hline
\end{tabular}

Table 4 Analysis of envelope peak value at postoperative complication

\begin{tabular}{|c|c|c|c|}
\hline & Absence & Presence & $P$ value \\
\hline R-peak value & $0.173 \pm 0.05$ & $0.218 \pm 0.06$ & 0.034 \\
\hline L-peak value & $0.173 \pm 0.06$ & $0.215 \pm 0.06$ & 0.048 \\
\hline Operated side peak value & $0.177 \pm 0.05$ & $0.218 \pm 0.08$ & n.p \\
\hline Air leakage & $n=41$ & $n=6$ & \\
\hline R-peak value & $0.173 \pm 0.05$ & $0.222 \pm 0.1$ & n.p \\
\hline L-peak value & $0.173 \pm 0.06$ & $0.225 \pm 0.06$ & 0.041 \\
\hline Operated side & $0.179 \pm 0.05$ & $0.229 \pm 0.09$ & 0.045 \\
\hline R-peak value & $0.173 \pm 0.05$ & $0.21 \pm 0.04$ & n.p \\
\hline L-peak value & $0.173 \pm 0.05$ & $0.196 \pm 0.05$ & n.p \\
\hline Operated side & $0.179 \pm 0.05$ & $0.196 \pm 0.05$ & n.p \\
\hline Non-operated side & $0.168 \pm 0.06$ & $0.21 \pm 0.04$ & n.p \\
\hline
\end{tabular}

FFT and high postoperative complication rate in those with high peak values using frequency analysis. The importance of measuring peak values is a new finding in this study. Furthermore, the increase in peak value in the complication group is thought to be due to the marked disturbance and discontinue of respiratory amplitude due to hypokinesia of the chest wall.

An important factor of our stretchable sensor is the ease of handling and high reproducibility. We measured the respiratory state preoperatively, which helped acquire the breathing pattern without noise. Therefore, abnormal respiratory patterns can be detected by creating and recognizing basic respiratory patterns.

In clinical practice, several healthcare professionals are involved in providing treatment. An alert function in such a sensor is promising in detecting deviations in the respiratory pattern. It can enable more universal observations of severe cases, which may support healthcare professionals with all levels of experience in providing postoperative care. Additionally, accumulation of data from actual patients is expected to expand the utility of this sensor to other areas, such as rehabilitation, evaluation of COPD, and respiratory 
evaluation of patients in the intensive care unit.

Identification of a pattern is important for clinical applications. In the future, we plan to analyze the wavelet and autocorrelation function method in addition to FFT.

\section{Limitations}

This study has several limitations. First, it involved a small number of patients with measurements performed only postoperatively from a single institution. Future collaborative research using larger sample sizes would help in obtaining accurate sensor measurements. Second, measurements were removed during noise, including during coughing and body movements and slacking or twisting of the sensor. Fourth, the effect of epidural anesthesia on chest wall movement has not been analyzed due to the small number of cases; therefore, the data may not represent the true respiratory patterns. Third, patient bias may be present in this feasibility study.

\section{Conclusions}

We investigated respiratory assessment using a stretchable sensor in postoperative management, which confirmed the feasibility of the sensor. It is promising in visualizing the respiratory state and detecting postoperative respiratory changes.

\section{Acknowledgments}

Funding: None.

\section{Footnote}

Reporting Checklist: The authors have completed the MDAR checklist. Available at http://dx.doi.org/10.21037/jtd-21156

Data Sharing Statement: Available at http://dx.doi. org/10.21037/jtd-21-156

Peer Review File: Available at http://dx.doi.org/10.21037/jtd21-156

Conflicts of Interest: All authors have completed the ICMJE uniform disclosure form (available at http://dx.doi. org/10.21037/jtd-21-156). The authors have no conflicts of interest to declare.
Ethical Statement: The authors are accountable for all aspects of the work in ensuring that questions related to the accuracy or integrity of any part of the work are appropriately investigated and resolved. This study was approved by the ethics committee of Tokyo Medical and Dental University (M2017-255), and informed consent was obtained from all patients. The study was conducted in accordance with the Declaration of Helsinki (as revised in 2013).

Open Access Statement: This is an Open Access article distributed in accordance with the Creative Commons Attribution-NonCommercial-NoDerivs 4.0 International License (CC BY-NC-ND 4.0), which permits the noncommercial replication and distribution of the article with the strict proviso that no changes or edits are made and the original work is properly cited (including links to both the formal publication through the relevant DOI and the license). See: https://creativecommons.org/licenses/by-nc-nd/4.0/.

\section{References}

1. Raviv S, Hawkins KA, DeCamp Jr M, et al. Lung cancer in chronic obstructive pulmonary disease: enhancing surgical options and outcomes. Am J Respir Crit Care Med 2011;183:1138-46.

2. Licker MJ, Widikker I, Robert J, et al. Operative mortality and respiratory complications after lung resection for cancer: impact of chronic obstructive pulmonary disease and time trends. Ann Thorac Surg 2006;81:1830-7.

3. De Torres JP, Bastarrika G, Wisnivesky JP, et al. Assessing the relationship between lung cancer risk and emphysema detected on low-dose CT of the chest. Chest 2007;132:1932-8.

4. Edwards JG, Duthie D JR, Waller DA. Lobar volume reduction surgery: a method of increasing the lung cancer resection rate in patients with emphysema. Thorax 2001;56:791-5.

5. Sato T, Watanabe A, Kondo H, et al. Long-term results and predictors of survival after surgical resection of patients with lung cancer and interstitial lung diseases. J Thorac Cardiovasc Surg 2015;149:64-70.

6. Mimae T, Suzuki K, Tsuboi M, et al. Surgical outcomes of lung cancer in patients with combined pulmonary fibrosis and emphysema. Ann Surg Oncol 2015;22:S1371-9.

7. Agostini P, Cieslik H, Rathinam S, et al. Postoperative pulmonary complications following thoracic surgery: are there any modifiable risk factors? Thorax 2010;65:815-818. 
8. Hahn G, Sipinkova I, Baisch F, et al. (1995). Changes in the thoracic impedance distribution under different ventilatory conditions. Physiological measurement 1995;16:A161.

9. Niu J, Shi Y, Cai M, et al. Detection of sputum by interpreting the time-frequency distribution of respiratory sound signal using image processing techniques. Bioinformatics 2018;34:820-7.

10. Hsu CH, Chow JC. Design and clinic monitoring of a newly developed non-attached infant apnea monitor. Biomedical Engineering: Applications, Basis and Communications 2015;17:126-34.

11. Tukanova K, Papi E, Jamel S, et al. Assessment of chest wall movement following thoracotomy: a systematic review. J Thorac Dis 2020;12:1031-40.

12. Suzuki K, Yataka K, Okumiya Y, et al. Rapid-response, widely stretchable sensor of aligned MWCNT/elastomer composites for human motion detection. Acs Sensors 2016;1:817-25.

Cite this article as: Kobayashi M, Wada Y, Okumiya Y, Yataka K, Suzuki K, Nakashima Y, Ishibashi H, Okubo K. Use of carbon nanotube sensor for detecting postoperative abnormal respiratory waveforms. J Thorac Dis 2021;13(5):3051-3060. doi: 10.21037/jtd21-156
13. Amjadi M, Kyung KU, Park I, et al. Stretchable, skinmountable, and wearable strain sensors and their potential applications: a review. Advanced Functional Materials 2016;26:1678-98.

14. Yang Y, Deng ZD. Stretchable sensors for environmental monitoring. Applied Physics Reviews 2019;6:011309.

15. Li L, Xiang H, Xiong Y, et al. Ultrastretchable fiber sensor with high sensitivity in whole workable range for wearable electronics and implantable medicine. Adv Sci (Weinh) 2018;5:1800558.

16. Kondo T, Uhlig T, Pemberton P, et al. Laser monitoring of chest wall displacement. Eur Respir J 1997;10:1865-9.

17. Al-Naji A, Gibson K, Lee S H, et al. Real time apnoea monitoring of children using the Microsoft Kinect sensor: a pilot study. Sensors 2017;17:286.

18. Cooper RM. Use and safety of percutaneous tracheostomy in intensive care Report of a postal survey of ICU practice. Anaesthesia 1998;53:1209-12. 